EP-184

\title{
Clinical review of characteristics on recurrence of resected pancreatic ductal adenocarcinoma
}

\author{
So Kyung YOON, So Jeong YOON, Ji hye JUNG, Sang Hyun SHIN, In Woong HAN, Jin Seok HEO* \\ Department of General Gurgery, Samsung medical center, Seoul, Korea
}

Introduction: Pancreatic ductal adenocarcinoma (PDAC) is a notorious disease famous for its high recurrence rate even after surgical resection with curative intent. The characteristics of the post-resection recurrence, which include patterns, location, timing and related factors, have not been clearly identified in previous studies.

Methods: We retrospectively analyzed medical data of the patients who underwent surgical resection with curative intent for PDAC between January 2007 and December 2016. The recurrence was defined as presence of either radiological or histological evidence, and the postoperative 1 year as a value differentiating between early and late recurrence groups.

Results: Among 732 patients, 555 patients (75.8\%) developed recurrence during the follow-up period. The 5-year overall survival rate and recurrence free survival of all patients was $28.9 \%$ and $19 \%$, respectively. Three hundred and fifty-eight patients (64.5\%) reccurred within the first year after surgery. Prognostic factors of early recurrence by univariate logistic regression analysis were significantly associated elevated CA-19-9 $(p=0.004)$, poorly differentiated tumor $(p<0.001)$, LN metastasis $(p=0.025)$ and preoperative tumor location (body to tail, $p=0.025)$. In the early recurrence group, metastasis occurred more in liver $(\mathrm{n}=116,30 \%)$ and in the late recurrence group, metastasis occurred more in lung $(n=37,21.8 \%)$ and local recurrence $(n=64,37.6 \%)$.

Conclusions: We have identified the characteristics of the post-resection recurrence. Further study to predict patients with high probability of early recurrence will help to establish individualized treatment. 